\title{
Real World Application of EBSD Forward Models
}

William Lenthe ${ }^{1}$, Edward Pang ${ }^{2}$, Michael Roach ${ }^{3}$, Jason Griggs ${ }^{3}$, Stuart Wright ${ }^{4}$ and Matthew Nowell ${ }^{5}$

${ }^{1}$ EDAX, United States, ${ }^{2}$ Department of Materials Science and Engineering, Massachusetts Institute of Technology, United States, ${ }^{3}$ School of Dentistry, University of Mississippi Medical Center, United States, ${ }^{4}$ EDAX, St Geprge, Utah, United States, ${ }^{5}$ EDAX, Riverton, Utah, United States

Pseudo-symmetry in electron backscatter diffraction (EBSD) - diffraction patterns with an apparent symmetry higher than the crystal symmetry - can significantly impact automated indexing algorithms when present. Pseudo-symmetry may arise for a variety of reasons including:

1. Small deviations from a higher symmetry super-group

o small lattice constant deviations e.g. a tetragonal material with c/a ratio near 1 or a triclinic material with $\alpha$ near $120^{\circ}$

O sub-lattice ordering (particularly of a higher $\mathrm{Z}$ element)

2. Special misorientations in some materials

○ MacKenzie cell vertices i.e. 2 n rotations about an $n$-fold axis

o low n $\Sigma$ n coincident site lattice misorientations

Pseudo-symmetry is present in a range of industrially relevant systems including steel, perovskites, and $\gamma$-TiAl [1,2]. Although the existence and scope of the problem is well established, resolving the correct orientation with traditional Hough indexing techniques has limited success [3-6]. The recent development of sophisticated dynamical EBSD simulations has shown new potential for predicting and resolving pseudo-symmetry [7-10].

$\mathrm{ZrO}_{2}$ is a promising material for dental implants that has monoclinic, cubic, and tetragonal phases with only slight distortions [11]. The tetragonal phase is nearly cubic with the double cell having a c/a ratio of 1.017. Master patterns of the Kikuchi sphere were simulated for each phase using EMsoft as shown in figure 1 [12]. Computing the normalized cross correlation between two master patterns for all rotations provides an estimate of phase discrimination difficulty [9]; a pair of master patterns with a maximum normalized cross correlation of 0 are trivial to distinguish while a maximum of 1 indicates the phases are identical.

A confusion matrix of maximum cross correlation is shown in figure 1 with peaks corresponding to symmetry operators removed for auto-correlation. The worst pseudo-symmetric misorientations for cubic, tetragonal, and monoclinc are 60 @ [111], 90 @ [110], and 180 @ [110] respectively. The results suggest the cubic and tetragonal phases of $\mathrm{ZrO}_{2}$ are difficult to distinguish and the tetragonal phase has significant potential pseudo-symmetry corresponding to a $45^{\circ}$ rotation about (110). Experimental results are consistent with the inability of Hough to discriminate cubic/tetragonal phases or resolve pseudosymmetry as shown in figure 2. Simulated and experimental results indicate distinguishing tetragonal patterns from cubic is harder than resolving the tetragonal pseudo-symmetry - potentially since the strain required to change the tetragonal $\mathrm{c}$-axis is larger than to transform from tetragonal to cubic. 


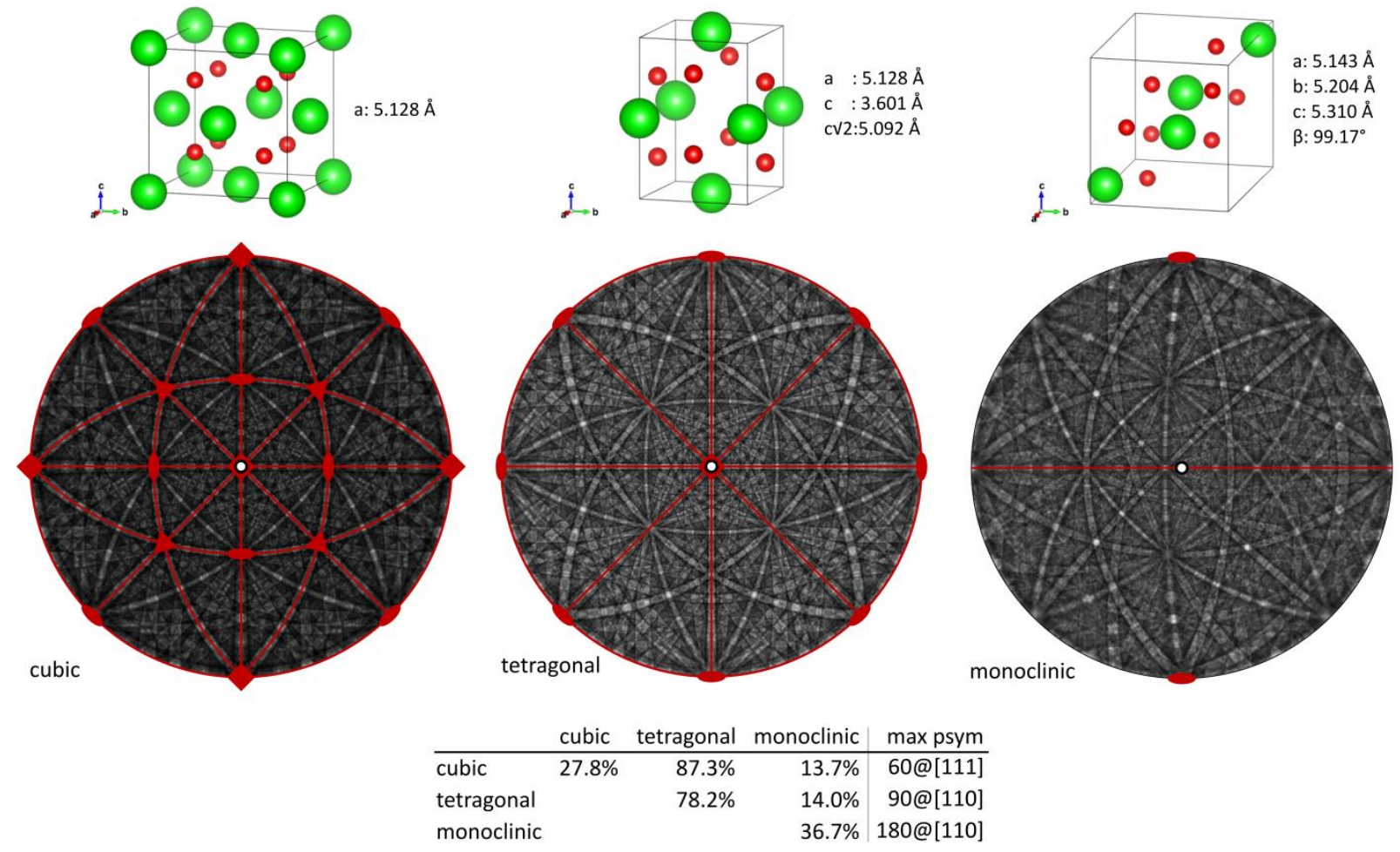

Figure 1. Unit cells (top) and master patterns (middle) are rendered for the cubic (left), tetragonal (center), and monoclinic (right) phases of $\mathrm{ZrO} 2$. The table shows the confusion matrix of normalized cross correlation between master pattern. Values from symmetry operators are excluded for auto-correlation with the maximum correlation misorientation in the right column. 


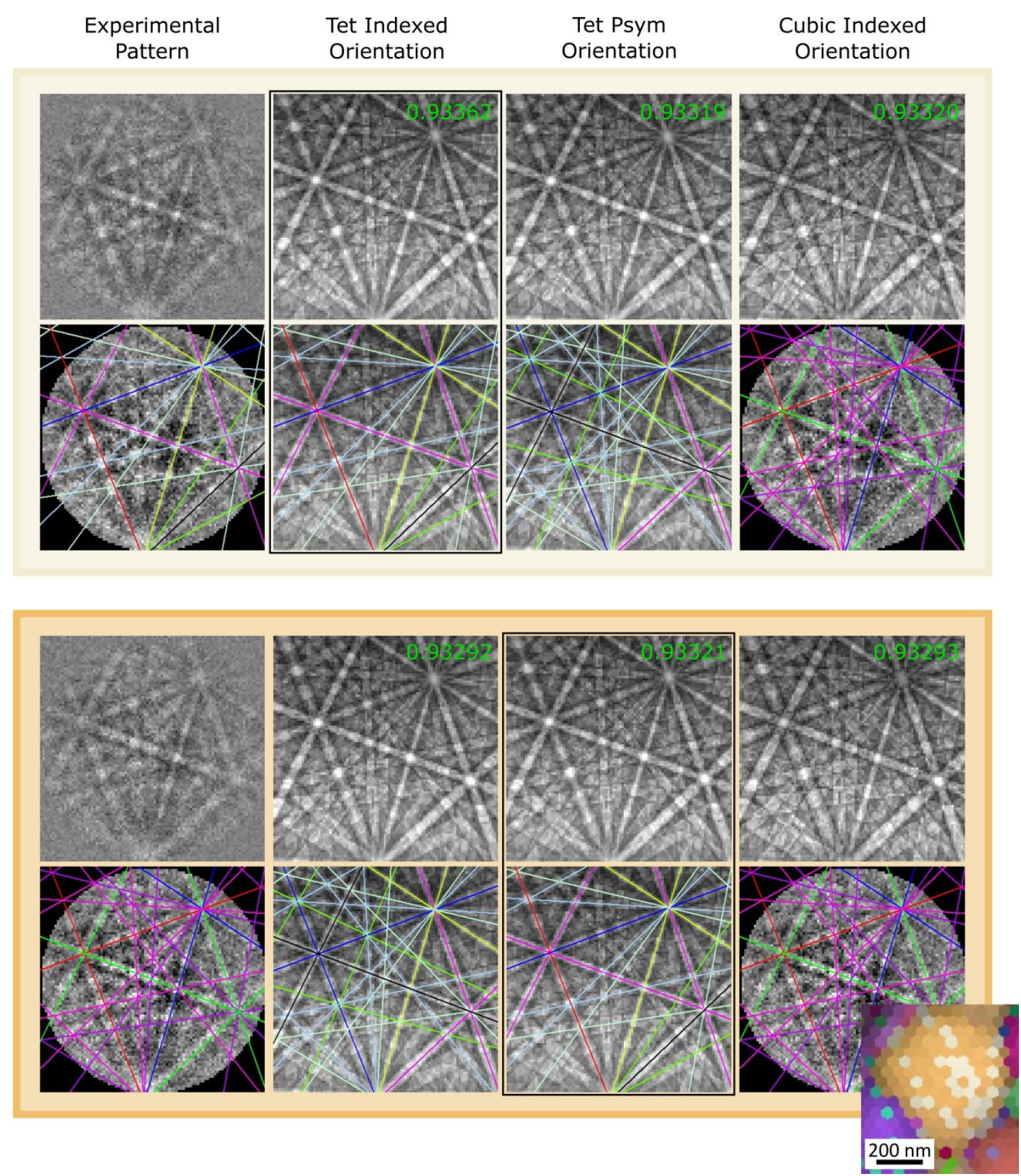

Figure 2. Hough indexing cannot resolve pseudo-symmetry for tetragonal $\mathrm{ZrO} 2 \mathrm{EBSD}$ patterns (far left column) resulting in a speckled IPF map (bottom right inset) yielding both the true (top half / light yellow) or pseudo-symmetric (bottom half / orange) orientations. The patterns for the true orientation are boxed with a black outline. Hough indexing also fails to distinguish the cubic result (far right column). Forward model based indexing correctly differentiates all 3 - normalized dot product between the experimental and simulated pattern is the green value in the top right of each pattern.

References

[1] Ryde, L. (2006). Materials Science and Technology, 22(11), 1297-1306.

[2] Jackson, B., Fullwood, D., Christensen, J., \& Wright, S. (2018). Journal of Applied Crystallography, 51(3), 655-669. 
[3] Zwart, P. H., Grosse-Kunstleve, R. W., Lebedev, A. A., Murshudov, G. N., \& Adams, P. D. (2008). Acta Crystallographica Section D: Biological Crystallography, 64(1), 99-107.

[4] Nowell, M. M., \& Wright, S. I. (2005). Ultramicroscopy, 103(1), 41-58.

[5] Abou-Ras, D., Gibmeier, J., Nolze, G., Gholinia, A., \& Konijnenberg, P. (2008). Crystal Research and Technology: Journal of Experimental and Industrial Crystallography, 43(3), 234-239.

[6] Zambaldi, C., Zaefferer, S., \& Wright, S. I. (2009). Journal of Applied Crystallography, 42(6), 10921101.

[7] Nolze, G., Winkelmann, A., \& Boyle, A. P. (2016). Ultramicroscopy, 160, 146-154.

[8] Marquardt, K., De Graef, M., Singh, S., Marquardt, H., Rosenthal, A., \& Koizuimi, S. (2017). American Mineralogist: Journal of Earth and Planetary Materials, 102(9), 1843-1855.

[9] Lenthe, W., Singh, S., \& De Graef, M. (2019). Journal of Applied Crystallography, 52(5), 1157-1168.

[10] Pang, E. L., Larsen, P. M., \& Schuh, C. A. (2020). arXiv preprint arXiv:2003.04476.

[11] Denry, I., \& Kelly, J. R. (2008). Dental materials, 24(3), 299-307.

[12] Singh, S., Ram, F., \& De Graef, M. (2017). Microscopy and Microanalysis, 23(S1), 212-213. 